\title{
Interactive comment on "In situ investigation of rapid subsurface flow: Identification of relevant spatial structures beyond heterogeneity" by C. Jackisch et al.
}

C. Jackisch et al.

conrad.jackisch@kit.edu

Received and published: 22 August 2016

We thank all five reviewers for their critical and constructive comments on our manuscript. We will carefully address all issues in the revision. In the general reply we provide a revised outline and an overview about the upcoming changes concerning this part of the companion MS. It is followed by detailed replies to and discussions of the comments of each review. 


\section{Consistency and generality of the presented experiments:}

The two companion MS deal with the in situ characterization of rapid subsurface flow. In order to highlight the methodological aspects of the study, we will refer to the general interplay of form and function. In our approach, we address this interplay through the detection of responses (MS1, function) and flow-relevant structures (MS2, form).

In accordance to MS1 a possible revised formulation for the title of this MS is: "Form and function in hillslope hydrology: In situ identification of flow-relevant structures".

We understand from the comments to both MS that the links between the different experiments, the measurements and the different aspects have not been conveyed as self-explanatory as anticipated. As suggested by the reviewers, we will revise both MS towards more clarity and self-consistency. The form - function reference shall help to clarify this.

Moreover, the lack of clear hypotheses caused substantial confusion. In accordance to the general frame, the MS at hand will primarily address form related issues along the following hypotheses:

H 2.1 Flow-relevant structures can be identified in the field under static conditions. (form described without function)

H 2.2 The characteristics of subsurface stormflow can be understood based on structural investigation. (form reveals function)

H 2.3 The localization of response patterns within the structural domain provides the missing link between form and function. (link form and function) 


\section{Revised outline of the MS}

Obviously, the structure of the MS requires revision in order to convey the key findings about the capabilities and limitations of multiple methods for subsurface structure identification at the pedon, plot and hillslope scale. Much confusion was caused by overlaps of process and structure related aspects. The revised MS will keep the focus on formrelated aspects and leave most of the process interpretation to the companion MS. This clearer focus will also make room for details about the pedon-scale exploration, which has been too brief before.

The upcoming revised MS will be outlined as follows:

1. Introduction

1.1 Form-function relationship in hydrological sciences and subsurface flow

1.2 General introduction about the identification of flow-relevant structures in the subsurface

1.3 Specific introduction including a brief summary about the headwater under study, the hypotheses, and the overall aims of the study

2. Methods

2.1 Local exploration

2.1.1 Sampling Design: Point measurements along catenas in nested sets around observation clusters during one campaign.

2.1.2 Used methods and respective scope, scale and capability

2.1.3 3D GPR survey of the hillslope

Printer-friendly version

2.2 Plot scale experiments

Discussion paper

2.2.1 Design: 3 close-by plot irrigations as repetitions with different intensity. 
2.2.2 Multi-method: Coherent use of dye and salt tracers, soil moisture monitoring, snap-shots of soil water composition regarding stable isotopes, time-lapse 3D GPR

2.3 Hillslope experiment

2.3.1 Design: Minimal replicate of plot scale setup + focus on lateral reaction on the hillslope. In conjunction with natural event to have an established connectivity and to reduce the effect of initial wetting and thus irrigation intensity and amount.

2.3.2 TDR network as spatially distributed reference. GPR transects as "virtual, noninvasive trenches".

\section{Results}

3.1.1 Point samples remain trapped in high heterogeneity but we can derive the mean integral plot properties (without its spatial organization).

3.1.2 3D GPR survey suggests a patchy layer of structures which later on prove to be not flow related. The driven case is needed.

3.2 Under dynamic conditions we see preferential flow and a lateral reaction in the deposit layer. But we cannot determine the hillslope response - especially as neither the share reaching the deposit layer nor the characteristics of the flow network can be determined.

3.3 TDR profiles are strongly limited in their spatial reference (resolution in time and space). GPR inferred trenches give a more comprehensive picture into the hillslope flow network as discrete, connected and leaky structures.

\section{Discussion}

4.1 Methodological discussions of the capabilities and limits of the used setups and methods

4.2 Conceptual discussion of heterogeneity versus structure 
5. Conclusions

H 2.1: No, the driven case is needed to identify flow-relevant structures.

H 2.2: Structural investigations (including irrigation experiments) can provide crucial information to understand the processes. However, structure alone does not reveal function.

Interactive

comment

$H$ 2.3: Form and function are mutually paired in the hydrological system. Overly strong assumptions about structures or processes can be avoided by the presented noninvasive GPR inferred trenching method, which can visualize and localize response patterns. This allows for more specific investigations and monitoring of subsurface processes.

\section{Overview about main upcoming changes}

In the specific replies to the reviews much more detail is given to all raised concerns. These changes will be embedded in the following revision schedule:

1. General story line and readability

1.1 Clarity about hypotheses, science questions and story line in abstract, intro, conclusions and rest of the MS.

1.2 Shortening where possible, extension where needed.

1.3 Clarity about the linkage between the methodological and case related aspects of the MS.

1.4 Final editing by a native English speaker.

2. Introduction

Printer-friendly version

2.1 strong revisions to refer to more studies around the globe

Discussion paper 
2.2 highlight the methodological aspects and refer to such works more specifically

2.3 link more clearly to the conceptual aspects of inferring hillslope-scale process understanding from local or signal measurements

2.4 clarify the used terminology and sketch of the form-function concept

3. Methods

3.1 Add the WHY to each aspect and draw their connection clearly

3.2 Add sampling design (especially of local exploration).

3.3 Add more details on the hillslope experiment (although repetitive to the companion MS).

3.4 Outline again how the methods will answer the overarching question of exploration of a structured subsurface.

4. Results

4.1 more details on local exploration

4.2 split case-related results from method-related ones

5. Discussion

5.1 Shorten discussion on case results and process interpretation to a minimum showing the limits and novelties of the results at the case of the upper Colpach basin.

5.2 Give explicit frame to methodological discussion to streamline the narrative towards the main findings.

6. Conclusions with reference to the hypotheses

Printer-friendly version

Many thanks again to all reviewers and Alex Zimmermann for your time and critique to substantially improve our MS.

Discussion paper 


\section{Reply to Review2}

General comments:

The authors present a rather comprehensive overview of results on the fast hydrological response of a hillslope to natural and artificial precipitation obtained by a series of complementary methods. As commented already by the first referee, the topic of the paper should be of quite some interest to the scientific community in this field. Nevertheless, I have some reservations about how the results are presented in the manuscript. I listed some general aspects below and subsequently added more detailed comments.

No clear hypotheses: Overall, the manuscript is predominantly of the type Look what we have found. It is a lengthy compilation of many (interesting) data without a clear hypothesis. Although the authors state clear research questions (L. $70-74)$, these questions are not consistently addressed. Neither the abstract nor the conclusions for example provide answers about the type of structures relevant for rapid subsurface flow and transport and how they can be identified. Simply stating that this transport takes place in specific structures (L. 593) does not really provide new insight. This deficiency is also obvious when reading the abstract, which mentions the challenges that have been faced and emphasizes the coherent combination of methods for identifying relevant structures and overall process understanding. What these structures and process understanding actually consist of is not made clear.

As addressed in the general reply, we see this deficit of clarity in the MS. The revised MS will refer to the concept of form and function. We formulate and address the following hypotheses to resolve the confusion:

$\mathrm{H} 1$ Flow-relevant structures can be identified in the field under static conditions. (form described without function)

$\mathrm{H} 2$ The characteristics of subsurface stormflow can be understood based on structural investigation. (form reveals function) 
$\mathrm{H} 3$ The localization of response patterns within the structural domain provides the missing link between form and function. (link form and function)

Moreover, we will restructure the MS and align the story line more clearly along these hypotheses.

Lack of generality: It is also hard (based on the manuscript) to generalise the presented findings beyond the very specific conditions at the field site (this aspects is also related to the problem mentioned above). What can be learned in general about preferential flow structures at hillslopes? What are general lessons about instrumentation for elucidating such structures that go beyond the recommendation Use several different methods at the same time on the same spot? This may sound overly critical but given the large effort such field experiments require (I very much appreciate this!) one should aim at gaining as much general insight as possible from such experiments.

The analyzed headwater is a specific example for the more general findings of the study. We will elaborate and emphasize the latter, which are of general interest to the community.

One of this is the critique to the standard approach of hydrological structural exploration based on field measurements and soil samples. We intend to highlight the conceptual limits when the highly heterogeneous subsurface is actually structured. In this case, also a higher number of samples will not help to identify structures. A second general finding is that flow-relevant structures require the response to an event to be revealed. In addition, this motivates the proposed non-invasive technique of time-lapse GPR to be able to identify flow-relevant structures without destroying the system under study. We also detail on the capabilities and limitations of this technique.

As such it is the proposed approach what we seek to generalize, not the specific exemplary findings about the upper Colpach basin. We feel that a lot of this has remained unclear due to the structural deficits of the MS. We will stress this methodological focus in the revised MS. Please consult the proposed outline in the general reply for details.

Printer-friendly version

Discussion paper 
Site description: Although the authors have described the site in the companion paper a minimum of information is also required in this manuscript. Even by consulting the companion paper, I could neither figure out the spatial location of the dye tracing experiments nor the date of the experiments (sec. 2.2). I also miss information on where the infiltration capacity measurements were taken (L. $104-108)$. This makes it very hard to properly interpret the results. The authors have to provide a similar map as in the companion paper locating all experiments and measurements relevant for this manuscript.

We agree with the reviewer and will address this by adding a more detailed description of the experimental design.

Experimental limitations: There are two aspects of the experimental procedure that struck me because they seem to (strongly) reduce the sensitivity of the experiments. First, the hillslope irrigation experiment was carried out briefly after substantial rainfall had been recorded $(43 \mathrm{~mm})$. This of course reduces the contrast of experimentally induced water content signatures. Additionally, the irrigation water was rather similar in its isotope signal (L. 385) as the soil moisture because it was stream water. In the end, this leads to a situation where part of the hillslope has received different isotopic input (irrigation plus precipitation) while the rest had received less water and only one type of isotopic signal (only precipitation).

It is true that the rainfall event prior to the hillslope experiment reduced the contrast in soil moisture (as the pre-experimental soil moisture was raised). The experiment was conducted one day after the natural event and thus at a time when the plot-scale advective flow had settled. With regard to activation of preferential flow structures the previous rainfall is considered as strength of the experiment. Through this the irrigation was suitable to initiate a new impulse of free water to drain in the subsurface preferential flow structures which was exactly desired by the experiment.

We agree that the use of isotopically enriched water would have increased the informa-

Printer-friendly version

Discussion paper 
tive value of the dataset. However, this was not possible due to practical reasons, and is discussed accordingly. The presented isotope data will be shifted to the companion MS, where it will be discussed in the context of the observed response dynamics.

This explanation will be added to the discussion of the hillslope experiment in the revised MS.

Fuzzy wording: The language is not very precise and often relies on a loose and vague use of words (see details below).

We will include a definition of the most important terms of structures, flow-relevant structures, response patterns and responses in the introduction and revise the MS to follow the nomenclature. We will furthermore make an extra effort for more precise wording. We will consult a native English speaker for final editing of the revised MS.

\section{Detailed comments:}

As the MS will be mostly rewritten, many of the comments may become obsolete. However, here are the replies which we will align the revisions on. Moreover, we have been challenged to relate the line numbers to the text as the reviewer must have used a differently compiled version of the MS. We hope to have related to the intended passages.

Title: The title is not very clear. What is meant by beyond heterogeneity?

We will change the title to avoid the confusing term. As this affects both companion papers, the final versions are still subject to discussion. A possible formulation for the title of this MS is: "Form and function in hillslope hydrology: In situ identification of flow-relevant structures". The discussion of heterogeneity vs. structure will be clarified in an appropriate discussion subsection.

Printer-friendly version

$\mathrm{L}: 14:$ What is a coherent combination?

Coherent means that the design gives sufficient overlap to really combine the findings

Discussion paper 
from different methods. This was found highly non-trivial as it involves a number of aspects from scale (in time and space) to technical issues (which measurements can be conducted jointly, which interfere each other). This will be clarified in the revised MS.

L. 28 - 37: What is about the use of GPR for characterising preferential flow paths? Given the topic of the manuscript l'd expect a short summary of which experiences have been obtained with regard to identifying flow paths and how they compare to results using different approaches.

The introduction will be revised to relate more clearly to studies using ERT or GPR for characterizing preferential flow paths. Infiltration experiments monitored by ERT have been also conducted in a neighboring headwater by M. Westhoff 2011 . There and in other studies decreases in resistivity underneath the irrigation site are shown. However, they can only explain a relatively small fraction of the total irrigation water. Moreover, the resolution in time and space is a mayor concern. From our study also GPR alone was not found capable to identify flow-relevant structures in the heterogeneous soil. Only a time-lapse application made such analyses possible. This opens another issue: The velocity and scale of the responses did not match the resolution of the GPR surveying. These methodological concerns will be detailed on specifically in the revised MS. As suggested by reviewer 1 we will also relate our findings in more detail to other studies like Guo et al. 2014 in the discussion.

L. 30: Flury et al., 1994, were probably the first to very systematically use dye tracers under standardised conditions in the field but they were for sure not the first to use dye tracers for staining preferential flow paths and infiltration patterns (see for example Steenhuis et al., 1990). Be careful when making absolute statements.

We will rephrase the sentence to: Dye staining has evolved as common practise since its first applications (presumably Bouma and Dekker, 1978) for a retrospective imaging of preferential flow paths. 
L. 31: Why are there strong assumptions involved when working with infiltration patterns caused by dye tracers? Be more explicit and precise.

Dye staining can only reveal an integral, a posteriori footprint of water which has passed certain sections of the soil and where dye has been resorbed to the matrix. It misses the shortest travel times, which either passed beyond the excavation depth or did not interact strongly enough to stain the flow paths. Another issue is that the temporal dynamics and initialization of flow paths remain indistinguishable. As such it does not reveal any information about the flow field characteristics alone. It may be even misleading the interpretation from high flows with low resorption being overlooked while patches of strong resorptions are overly emphasized. With regard to tracer breakthrough studies (e.g. into tile drains) only the short travel times can be observed and the slow phase is missed. Hence it is very desirable to develop in situ methods which are capable to capture the whole spectrum of flow processes including its spatial arrangement.

The revised MS will separate the general structural exploration from the identification of flow-relevant structures. With respect to the findings of the plot-scale irrigation experiments we will detail on the interesting combination of the results from dye staining and 3D GPR. This will also include a more detailed discussion of assumptions concerning these methods.

\section{40: What are our current theories? What do they predict?}

The main message was not meant to focus on the 'current theories', but on the limited availability of experimental data. Because we cannot see into the subsurface, there are only very few examples of investigations in the real world. In contrast a large number of proposed models is used in hydrological sciences to explain and understand subsurface stormflow in soil structures. Hence, the presented methods to non-invasively reveal subsurface flow would be a strong asset to advance hydrological sciences. The sentence will be rephrased to convey this message. 
L. 47: What have these model studies shown in a successful manner? Be more clear what you mean.

We intended to have summarized this in the given list of citations. To be more precise, the formulation will be changed to:

Although such model studies have been very successful in replicating the behavior of the modelled system (Superflex in the same basin as this study reveals a suitable model structure to reproduce the runoff behavior (Fenicia et al., 2014); Catflow could reproduce an irrigation experiment at the hillslope-scale by introduction of cells with elevated conductivity in the hillslope grid (Klaus and Zehe, 2010); Vertical tracer translocation could be predicted based on explicit 3D structure representation (Vogel et al., 2006); and many more), they require strong assumptions about connectivity and interaction between matrix and structures (Gerke, 2006).

L. 51 - 52: I am surprised by this statement. Many experiments have been carried out for studying preferential flow and the exchange with the surrounding matrix. Can you specify?

We aim to highlight the process perspective here. With respect to the large body of literature on soil water transport in heterogeneous soils, the investigations of preferential flow patterns are already few. Within this field most studies reveal the preferential flow paths (e.g. by dye staining) but cannot detail on the process of macropore-matrixinteraction from an experimental perspective. This links back to the intention to propose a method to non-invasively study such responses with the aim to hopefully generate more evidence about the main controls of stormflow characteristics.

However, as this aspect of the introduction points strongly to the process domain (which is the topic of the companion MS), we will reduce or even drop this aspect in the revised MS for clarity.

L. 61: This sentence evokes the impression that people mostly care about the numeri- 
cal aspects of model quality. I don't think that is true.

This impression was not intended. The issue we try to point out is, that the perceptual models have a strong influence on the final models. At the same time these models require specific parameters which are more coherent to the perceptual model than to the respective system under study. As models are intended as abstraction of the systems to the minimal adequate level, we propose that in situ imaging of subsurface flow processes will have a high benefit to close the gap between the modelers' and experimentalists' concerns. This will be elaborated more clearly.

L. 62 - 63: Which ambiguity of measurements do you refer to here?

This relates to the representativeness of point measurements for structural exploration and monitoring. We will provide more theoretical context to prepare one of the key findings of our study that without being set into context such measurements are insufficient to reveal subsurface flow structures and even susceptible to erroneous conclusions.

L. 71: Here you conceptually switch from fast (solute) transport (= preferential flow) to fast hydrological responses, which do not necessarily imply preferential flow. You have to conceptually separate these phenomena and make this explicit throughout the entire manuscript. This has to start in the Introduction and go all the way to the results and discussion: Which of the approaches actually reveal fast transport, which may reflect also a fast discharge of pre-event water?

We will revise the MS accordingly and include a definition of the most important terms in the introduction. This will also include how we see the experiments and findings with respect to celerity and velocity.

L. 80: What do you mean by framing local heterogeneity? L. 80: What is rather unknown?

In general terms, heterogeneity can be seen as deviation of the found reality from the concept of quasi-homogeneous entities. If this deviation concerns only the apparent 
values of the same physical process, more samples are adequate to determine its stochastics. In cases (like here) where this deviation also means a shift in the physical processes, heterogeneity becomes a scale-problem. Here any measurement will consist of an unknown subset of connected or not connected flow paths. This makes it impossible to unravel the properties of the different flow domains without knowing the composition of the explored ensemble of each measurement. Hence the techniques cannot determine the sub-scale (with respect to the measurement footprint) organization of the domain and will only recover heterogeneity independent of the number of samples.

We will rephrase the sentence to: We started with a large number of hydrological field and laboratory measurements, which eventually only displayed local heterogeneity without revealing characteristic structures. Similarly, spatially distributed but static methods, like 3D GPR surveying, revealed some subsurface formations but no coherent structures which would allow to characterize the hillslope. Moreover, the issue will be discussed more clearly in the revised MS.

L. 82: What do you mean by driven conditions? I assume you refer to experimentally controlled boundary conditions, is this right?

The driven case describes the situation, when the system is deviating from local thermodynamic equilibrium due to the input of mass (water). We will detail on the used terminology in the revisions and consider "dynamic conditions" as alternative formulation.

L. 86: What do you mean by coherently combining multiple methods? How is this achieved and differs from incoherent combinations of methods?

We intended to highlight the challenge to combine multiple methods in a way that the same response is recorded by multiple techniques on multiple scales. The measurements have different resolution and operate on different assumptions. As coherent combination we see measurements which can test each other's perception. In the re-

Printer-friendly version

Discussion paper
Interactive

comment 
visions we will clarify this by providing more details on the issue of the experimental setups and by emphasizing the actual story line about structural exploration.

L. 134: Why have two irrigation rates been used? What are the consequences? Discuss.

One of the objectives of the experiments has been the characterization of the advective flow field at the site. Because of this, it was necessary to also check how much the recorded flow field changes with different irrigation intensities. As the focus of the MS is on the exploration of flow-relevant structures, we neglected the discussion of the process dynamics. We will include this reasoning in the discussion of the revised MS and detail on the implications.

\section{213 - 214: In order to achieve what?}

I assume this relates to the determination of the spatial extent and irrigation intensity of the hillslope experiment through a priory application of the 2D process model CATFLOW (P7L4): The hillslope experiment extends the plot experiments in scale. The primary question was what irrigation impulse shall be selected for an activation of flow paths along the hillslope. A secondary question was what spatial monitoring extend is required to monitor the hillslope reaction. The model runs were based on data from the local exploration assuming periglacial deposit layers as conductive layers in the hillslope. In a series of scenarios, the one with $30 \mathrm{~mm} / \mathrm{h}$ irrigation for $4 \mathrm{~h}$ turned out to be well balanced. Moreover, a reaction in the subsurface about $10 \mathrm{~m}$ downslope was anticipated. This was then used to design the experiment after. The formulation will be revised to clarify these aspects.

L. 220: One cannot see that in Fig. 7. Only Fig. 2 in the companion paper makes this clear.

Printer-friendly version

We will add a figure outlining the experimental setup as well as a map including all measurements and experimental sites. Figure 7 will be changed to contain only information

\section{C16}

Interactive

comment
Discussion paper

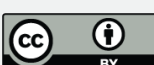


specific to the 3D GPR survey results and the identified potential structures.

L. 241: Sorry, it is not clear to me what was actually done here.

I assume this relates to P7L25ff. The issue of interpolation and resampling arises from the necessity to synchronize the different singular measurements, which were taken at different times by the same probe. To do so, we generated an intermediate grid of high data density onto which linearly interpolated versions of the time series are projected. We then resample from the intermediate grid to derive a synchronized version of the records. We will clarify this in the revisions.

L. Sec. 2.3.5: This paragraph is confusing. Why did you decide to run the experiment so shortly after $43 \mathrm{~mm}$ of rain? This implies that the soil was already wet and not much additional change can be expected.

The paragraph will be revised containing a clarification why the preceding rainfall is beneficiary to the experiment and what implications arise from it. Please also see the reply to the general comment above.

L. 306: What is meant by strong reaction?

The recovered tracer masses peak in this depth. The wording will be changed to be more precise: Tracer recovery peaks in a depth of approximately $0.6 \mathrm{~m}$ in all plots. Soil moisture change is strongest in $0.5 \mathrm{~m}$ and $0.7 \mathrm{~m}$ depth in plot $X$ and $X I I$ respectively.

L. 310: Which is plot XI? Please provide a proper map, where such information is provided.

The three plots are in direct proximity with about $1.5 \mathrm{~m}$ distance between them. A map describing sampling design and locations in detail will be included in the revised MS.

L. 312: What are stronger interactions with the soil matrix?

In comparison to plot XI and XII, the matrix adsorbed much more tracer in plot X. Most likely this is due to some disturbance of the soil by perturbation (eg. a fallen tree 
nearby). We will clarify this in the revisions.

Fig. 3: Please provide units for the grid. What is the physical meaning of negative volumetric water content? This makes only sense if the units represent change of water content over time. Please correct the units accordingly. From the pictures it seems that there was quite some microtopography affecting the infiltration patterns. How relevant is that for the subsequent interpretation?

1. The grid: The tracer recovery grid in the second column uses the sample column as $x$ axis unit. The grid is $5 \times 5 \mathrm{~cm}$ as given in the caption. We will change the figure to contain meters as unit.

2. Changes in soil moisture: Sorry for the unclear heading. It will be changed to $\mathrm{d}$ (theta) $\left[\mathrm{m}^{3} / \mathrm{m}^{3}\right]$ with the subtitle soil moisture change over time. As the plots show absolute changes in soil moisture the units are correct. We will add them to the colorbar for clarity. The given soil moisture change can become negative when the recorded value falls below the initial one.

3. Microtopography: Microtopography is less an issue than funneling in the litter layer. So far we did not separate this effect from the overall imprint of flow-relevant structures. We agree that this may be a critical issue and will include it in the discussion of the experiments.

L. 324: Slight correlation: please be quantitative and provide numbers.

We will calculate some goodness of fit measures in the revisions although the number of points is rather low and it is more a pattern comparison than a correlation analysis.

L. 343: Please show results for plot $X$ (possibly in the SI).

We opted to show only the plot XI and XII because of three reasons: 1) We wanted to not exceed the number of figures even more. 2) The acquisition timing at plot $X$ has been longer than for the other ones. This results in a stronger blur of the resulting similarity attributes. 3) As pointed out earlier and shown in figure 3, macropore-matrix- 
interaction has been stronger at plot $X$ which makes the plot less comparable to the other two, where the difference is mostly limited to the irrigation intensity. We can include the figure as supplement.

Fig. 395: How can you distinguish between event and irrigation water with GPR?

The procedure is described in detail in section 2.3.5. As the description exceeds the frame of the figure caption, we will keep the current form and add a reference to the respective text section in the figure caption instead.

L. 409: So what was your expectation? You never mentioned this before? Did you not know that there was no strong subsurface reflector based on your previous (pedological) investigations?

I suspect this relates to P13L9: The pedological exploration actually expected the deposit layer to give at least some traceable contrast in the radargrams. But this expectation was proven wrong. In the revised MS the separation between exploration of pure structure and of flow-relevant structures will be made. This will include more details about the 3D survey at the hillslope and the difficulty to identify any kind of structure in the heterogeneous reflectors.

L. 425 - 427: What are these common assumptions - that there are two flow domains that are completely separated? The fact that preferential flow paths end somewhere in the soil matrix is not new (just look at some of the infiltration patterns in Flury et al., 1994, Kulli et al., 2003, or Weiler (2005)).

We agree that the challenge of non-isolated multiple flow domains is not new. Current models for preferential flow came up with a number of suggestions how to include it. This is summarized in the introduction P2L22ff. The observed signal in the hillslope experiment is not merely conveyed through the system (as would be the case for no leakage) but decays strongly with distance to the irrigation core area. For the characterization of the flow-relevant structure this is an important fact although its pa- 
rameterization remains challenging. This aspect will be addressed more clearly in the revised MS.

Interactive comment on Hydrol. Earth Syst. Sci. Discuss., doi:10.5194/hess-2016-190, 2016.

Interactive

comment 